TRANSACTIONS OF THE

AMERICAN MATHEMATICAL SOCIETY

Volume 174, December 1972

\title{
TOPOLOGIES OF CLOSED SUBSETS
}

BY

\section{LOUIS NARENS $\left({ }^{1}\right)$}

ABSTRACT. In this paper various topologies on closed subsets of a topological space are considered. The interrelationships between these topologies are explored, and several applications are given. The methods of proof as well as some intrinsic definitions assume a familiarity with A. Robinson's nonstandard analysis.

E. Michael (Topologies of spaces of subsets, Trans. Amer. Math. Soc. 71 (1951), 152-182), K. Kuratowski (Topology, Vols. I and II, Academic Press, New York, 1968), L. Vietoris (Beriche zweiter Ordnung, Monatsh. Math.-Phys. 33 (1923), 49-62), and others have considered methods of putting topologies on closed subsets of a topological space. These topologies have the property that if the underlying topological space is compact then the topology of closed subsets is also compact. In general, however, these topologies of closed subsets are not compact. In this paper, a topology of closed subsets of a topological space is constructed that is always compact. This topology is called the compact topology and has many pleasant features. For closed subsets of compact Hausdorff spaces, this topology agrees with Vietoris' topology. For arbitrary spaces, there are interesting connections between the compact topology and topological convergence of subsets, including generalized versions of the Bolzano-Weierstrass theorem.

1. Preliminaries. Throughout this paper topologies are specified by giving a set together with its closed subsets. Thus, if $(X, \Gamma)$ is a topological space, then " $X$ " will denote a set and " $\Gamma$ " will denote the family of closed subsets of $X$. If $(X, \Gamma)$ is a topological space and $A \subset X$ then $A^{c}$ will denote the closure of $A$, and $A^{\sim}$ the complement of $A$ in $X$.

We will basically follow A. Robinson's treatment of topological spaces that is given in [8]. (The symbol " $\simeq$ ", however, has a specialized use.) Among the more important concepts of nonstandard analysis that will be used in this paper are the following:

(1) Concurrent relations. The relation $R(x, y)$ is said to be a concurrent relation if and only if for each finite set $A$, if for each $a \in A$ there is a $y$ such that $R(a, y)$, then there is a $z$ such that for each $a \in A, R(a, z)$.

Received by the editors September 1, 1970.

AMS (MOS) subject classifications (1970). Primary 02H25, 54A20, 54J05; Secondary $54 \mathrm{~A} 10$.

Key words and phrases. Nonstandard analysis, topologies on spaces of sets, topological convergence of sequences of sets, generalized Bolzano-Weierstrass theorem.

(1) This paper represents a portion of the author's Ph. D. dissertation, which was prepared under the supervision of Professors A. Robinson and Y. Moschovakis at the University of California at Los Angeles. The author wishes to express his gratitude to Dr. Perry Smith for his many suggestions and corrections of earlier versions of this paper. The preparation of this paper was supported in part by NSF Grant No. GP-8827. 
(2) Enlargements. Let $\mathfrak{A}$ be a full mathematical structure. That is, $\mathfrak{O}$ is a higher order mathematical structure that is composed of objects of type $i$ for $i=$ $0,1,2, \cdots$. The objects of type 0 are the elements of a given set. The objects of type $i>1$ are all possible $n$-ary relations between objects of lower type. Let $\mathbf{L}$ be a language that describes $\mathfrak{A}$. We assume that $\mathrm{L}$ has constant symbols corresponding to each element of $\mathfrak{U}$. Let $L^{\prime}$ be the language $L$ together $w$ ith a new constant symbol $a_{R}$ corresponding to each concurrent relation $R$ in 2 . Let the set of sentences, $\mathcal{E}$, of $L^{\prime}$ be defined as follows: $R\left(a, a_{R}\right) \in \mathcal{E}$ if and only if $R$ is a concurrent relation in $\mathfrak{U}$ and $a$ is an element of $\mathfrak{U}$ that is in the domain of $R$. Of course, $\mathbf{R}(\mathbf{x}, \mathbf{y})$ is the relation in $\mathbf{L}$ that corresponds to $R$ and $\mathbf{a}$ is the constant symbol that corresponds to $a$. Let $\mathcal{F}$ be the set of true sentences of $\mathcal{U}$ in the language $L$. Then $\mathcal{F} \cup \mathcal{E}$ is a consistent set of sentences in the language $L^{\prime}$, and an enlargement of $\mathcal{U}$ is a Henkin model of the sentences $\mathcal{F} \cup \mathcal{E}$. In particular, an enlargement of $\mathfrak{U}$ is an elementary extension of $\mathfrak{U}$. In this discussion, $\mathfrak{U}$ is called the standard model.

(3) * -notation. Let $\mathfrak{U}$ be a standard model and ${ }^{*} \mathfrak{U}$ an enlargement of $\mathfrak{U}$. If $A$ is an object in $\mathscr{U}$ then ${ }^{*} A$ is the object in ${ }^{*} \mathcal{U}$ that corresponds to the constant symbol $\mathbf{A}$ in the language $\mathbf{L}$. If $B$ is an element of ${ }^{*} \mathcal{U}$, and for some $A$ in $\mathfrak{U}, B={ }^{*} A$, then $B$ is said to be standard. (Sometimes the word "standard" will be used redundantly for emphasis.)

(4) Monads. Let $\mathfrak{Q}$ be a standard model of a topological space, * $\mathfrak{U}$ an enlargement of $\mathfrak{A}$, and $x$ be a point in $\mathfrak{X}$. Then the monad of $x$ is $\bigcap_{U \in \Delta}{ }^{*} U$ where $\Delta$ is the family of open subsets in the standard model that contain the point $x$. If $a$ is a standard point, and in the enlargement, $b$ is in the monad of $a$, we write $a \simeq b$. Note that by this definition, $a \simeq b$ implies that $a$ is a standard point. However, if the topological space is Hausdorff, then the monads of distinct standard points have empty intersection, and it will be convenient to express that $x$ and $y$ are in the same monad in this case by writing $x \simeq y$. If $x$ is a point in ${ }^{*} \mathscr{U}$ and $x$ is in the monad of some standard point then $x$ is said to be near-standard. By a fundamental theorem of A. Robinson, a topological space is compact if and only if in every enlargement of the space each point is near-standard.

Since compactness and near-standardness are intimately related, an intriguing possibility arises: If one could define a relationship of "near-standardness" among objects of a standard set, ${ }^{*} A$, in the enlargement in such a way that each object in " $A$ is "near-standard," then one might be able to define a compact topology on $A$ in the standard model by using this notion of "near-standardness." For example, let $X$ be the set of points of a topological space and $\Delta$ the family of all closed subsets of $X$. Then in the enlargement we want to define a relationship of "near-standardness" among the members of " $\Delta$. That is, if $A \epsilon^{*} \Delta$ we want to find a ${ }^{0} A \in \Delta$ such that ${ }^{*}\left({ }^{0} A\right)$ is "near-standard" to $A$. One obvious possibility is that 
${ }^{0} A=\{x \in X \mid x \simeq y$ for some $y \in A\}$. In $\$ 3$ we will see that this does indeed define an interesting compact topology on $\Delta$. However, a technical difficulty arises: We must be able to verify that if $A \in{ }^{*} \Delta$ then ${ }^{0} A$ is a closed subset in the standard model. If $A$ is standard, this is always the case. A. Robinson has shown in [8, Theorem 4.3.12] that if the standard model is a first countable space then ${ }^{0} A$ is closed. For arbitrary topological spaces, however, a model more powerful than an enlargement is needed to show this. In the next section this model is constructed. Since this is the only result of $\$ 2$ that is used in the subsequent sections, the reader may skip the lengthy construction on the first reading of this paper.

It should be noted that W. A. J. Luxemburg in [5, Theorem 3.4.2] constructs a model (by a different method than in $\$ 2$ ) in which ${ }^{0} A$ is always closed. By using the methods of $\mathrm{H}$. J. Keisler, an internal set, $A$, of an enlargement can be given which is such that ${ }^{0} A$ is not closed. (See Example 3.4.3 in [5].)

2. Special enlargements. Let $X_{0}$ be a topological space and $\mathfrak{U}_{0}$ a model of $X_{0}$. For each natural number $n$, let $\mathfrak{U}_{n+1}$ be an enlargement of the structure $\mathfrak{U}_{n}$. Let $\mathfrak{A}_{\omega}=\bigcup_{n \in \omega} \mathfrak{A}_{n}$, where $\omega$ is the set of natural numbers. Then $\mathfrak{A}_{\omega}$ is an enlarge ment for $\mathfrak{U}_{i}$ for each $i \in \omega .\left({ }^{2}\right)$

Definition 2.1. $\mathfrak{X}_{\omega}$ is called a special enlargement of $\mathfrak{A}_{0}$.

Notation. Since for each $i \leq \omega, \mathfrak{A}_{i}$ is a model of a topological space, let $X_{i}$ be the (internal) topological space of $\mathfrak{U}_{i}$. That is, let $X_{i}$ be the extension of $X_{0}$ in the model $\mathfrak{U}$.

Notation. Suppose that $V \subset X_{i}$ for some $i \in \omega . V$ need not be an internal subset of $X_{i}$ in the model $\mathfrak{U}_{i}$. Since $\mathfrak{U}_{i+1}$ is an enlargement of the structure $\mathfrak{U}_{i}$, there is an internal subset of $X_{i+1}$ in the model $\mathfrak{U}_{i+1}$ that corresponds to $V$. This internal subset is denoted by ${ }_{i} V$. Thus if $U$ is a subset of $X_{0},{ }_{0} U$ is the name of the set that corresponds to $U$. This correspondence, of course, depends upon the model. Thus in $\mathfrak{U}_{0},{ }_{0} \mathrm{U}$ corresponds to $U$, while in $\mathfrak{U}_{1},{ }_{0} U$ is the name of some set $V$ that is the extension (in $\mathscr{U}_{1}$ ) of $U$. Note that if $V_{0}, V_{1}, \cdots, V_{\omega}$ are the interpretations of ${ }_{0} \mathrm{U}$ in $\mathfrak{U}_{0}, \mathfrak{U}_{1}, \cdots, \mathfrak{U}_{\omega}$ respectively, then $V_{0} \subset v_{1} \subset \cdots \subset V_{\omega}$.

Once again, let $U$ be a subset of $X_{0}$. Since $U \subset X_{1},{ }_{1} U$ is a name of a set $V$ that is an internal subset of $X_{2}$ in the model $\mathfrak{A}_{2} . V$ is, of course, an extension (in $\mathrm{P}_{2}$ ) of $U$. The relationship of ${ }_{0} U$ and ${ }_{1} U$ is given in the following theorem:

Theorem 2.1. If $U \subset X_{0}$ then $\mathfrak{H}_{2} \vDash{ }_{1} \mathrm{U} \subset{ }_{0} \mathrm{U}$.

Proof. It is true about the model $\mathfrak{U}_{1}$ that " $U$ is a subset of ${ }_{0} U$." Since $\mathfrak{U}_{2}$ is an enlargement of the structure $\mathfrak{U}_{1}, \mathfrak{U}_{2}$ preserves this truth. In the language appropriate to $\mathfrak{U}_{2}$, this says that " $\mathrm{U} \subset{ }_{0} \mathrm{U}$."

( ${ }^{2}$ ) Abraham Robinson in Compactification of groups and rings and nonstandard anal$y$ sis (in J. Symbolic Logic 34(1969), 576-588) also uses the union of a denumerable sequence of enlargements to elicit certain compactification properties. 
Similarly, one can in general show that if $U \subset X_{0}$ then $\mathfrak{U}_{i+1} \vDash_{i} \mathbf{U} \subset{ }_{0} \mathbf{U}$. Then by using elementary equivalence between models, the following theorem can easily be shown:

Theorem 2.2. If $0 \leq i<j \leq \omega$ and $U$ is a subset of $X_{0}$ then $\mathfrak{d}_{j} \vDash{ }_{\mathrm{i}} \mathrm{U} \subset{ }_{0} \mathbf{U}$.

Definition 2.2. If $x \in X_{0}$ and $i \leq \omega$, then $\mu_{i}(x)$, the monad of $x$ in $\mathfrak{U}_{i}$, is defined as follows: $y \in \mu_{i}(x)$ if and only if for each open subset $U$ of $X_{0}$, if $x \in U$ then $\mathscr{Q}_{i} \vDash y \in{ }_{0} \mathrm{U}$. Note that if $i<j \leq \omega$ and $\mathscr{U}_{i} \vDash y \in{ }_{0} \mathrm{U}$ then $\mathfrak{U}_{j} \vDash y \in{ }_{0} \mathrm{U}$. Thus if $i<j \leq \omega$ then $\mu_{i}(x) \subset \mu_{j}(x)$.

Definition 2.3. If $A$ is a subset of $X_{\omega}$, let

$$
{ }^{0} A=\left\{x \in X_{0} \mid \mu_{\omega}(x) \cap A \neq \emptyset\right\} \text {. }
$$

Definition 2.4. Let $\mathrm{S}(V)$ be the predicate that says " $V$ is a subset of $X_{0}$."

Theorem 2.3. If $\mathfrak{Q}_{\omega} \vDash \mathbf{S}(\mathbf{A})$ and $\mathbf{A}$ is a name of the set $A$ in the model $\mathfrak{O A}_{\omega}$, then ${ }^{0} A$ is a closed subset of $X_{0}$.

Proof. Suppose that $\mathfrak{A}_{\omega} \vDash \mathbf{S}(\mathbf{A})$ and $\mathbf{A}$ is a name of the set $A$ in $\mathfrak{U}_{\omega}$. Since $\mathfrak{H}_{\omega}$ is a union of a chain of models, there is an $i \in \omega$ such that $\mathfrak{A}_{i} \vDash \mathrm{S}(\mathrm{A})$. Let $x$ be an accumulation point of ${ }^{0} A$. If $U \subset X_{0}$, let $U^{\prime}$ be the interpretation of ${ }_{0} U$ in $\mathfrak{A}_{i}$. Let $A^{\prime}$ be the interpretation of $\mathbf{A}$ in $\mathfrak{U}_{i}$. Let $R(U, y)$ be the following relation:

$$
\begin{aligned}
& \text { " } U \text { is an open subset of } X_{0} \text { and } x \in U \text { ' } \\
& \text { and } y \in U \text { ' and } y \in A \text { '." }
\end{aligned}
$$

Then $R(U, y)$ is a concurrent relation on $\mathfrak{A}_{i}$. For suppose that $U_{1}, \cdots, U_{n}$ are such that $\exists y_{1} R\left(U_{1}, y_{1}\right)$ and $\cdots$ and $\exists y_{n} R\left(U_{n}, y_{n}\right)$. Let $U=\bigcap_{1 \leq j \leq n} U_{j}$. Then $U$ is an open subset of $X_{0}$ and $x \in U$. Since $x$ is an accumulation point of ${ }^{0} A$, $\mathscr{U}_{\omega} \vDash_{0} \mathbf{U} \cap \mathbf{A} \neq \emptyset$. By elementary equivalence, $\mathfrak{U}_{i} \vDash_{0} \mathbf{U} \cap \mathbf{A} \neq 0$. Therefore, there is a $y$ such that $\mathfrak{U}_{i} \vDash\left(x \epsilon_{0} \mathbf{U}\right.$ and $y \epsilon_{0} \mathbf{U}$ and $\left.y \in \mathbf{A}\right)$. In other words, $x \in U^{\prime}, y \in U^{\prime}$, $y \in A^{\prime}$. Since for $j=1, \cdots, n, U \subset U_{j}, R\left(U_{j}, y\right)$ holds.

Since $R(U, y)$ is a concurrent relation on the structure $\mathfrak{U}_{i}$ and $\mathfrak{U}_{\omega}$ is an enlargement of $\mathscr{U}_{i}$, there is a $y_{0}$ such that for all open subsets, $U$, of $X_{0}$ such that $x \in U, \mathfrak{A}_{\omega} \vDash R\left({ }_{\mathrm{i}} \mathbf{U}, y_{0}\right)$. Therefore by Theorem 2.2, $\mathfrak{U}_{\omega} \vDash\left(x \in{ }_{0} \mathbf{U}\right.$ and $y_{0} \in{ }_{0} \mathbf{U}$ and $\left.y_{0} \in \mathbf{A}\right)$. Since this is true for each open subset $U$ of $X_{0}$ such that $x \in U$, $y_{0} \in \mu_{\omega}(x)$ and $y_{0} \in A$. Hence $x \in{ }^{0} A$.

3. The compact topology. Throughout the rest of this paper we will assume that all enlargements are special enlargements.

Let $(X, \Gamma)$ be a topological space. (Recall that $\Gamma$ is the family of closed subsets of $X$. ) If $A \epsilon^{*} \Gamma$ let

$$
{ }^{0} A=\{y \in X \mid \text { there is an } x \in A \text { such that } y \simeq x\} \text {. }
$$

By Theorem 2.3, ${ }^{0} A \in \Gamma$.

Theorem 3.1. If $A \in \Gamma$ then ${ }^{0}\left({ }^{*} A\right)=A$. 
Proof. Assume $A \in \Gamma$. Let $x \in A$. Then $x \epsilon^{*} A$. Since $x$ is standard, $x \in{ }^{0}\left({ }^{*} A\right)$. Therefore, $A \subset{ }^{0}(* A)$. Let $x \in{ }^{0}(* A)$. Then there is a $y \in \epsilon^{*} A$ such that $y$ is in the monad of $x$. Let $U$ be an arbitrary standard open set with $x \in U$. Since $y$ is in the monad of $x, y \in{ }^{*} U$. Therefore, ${ }^{*} U \cap{ }^{*} A \neq \emptyset$. By elementary equivalence, $U \cap A \neq \varnothing$. Thus $x$ is an accumulation point of $A$. Since $A$ is closed, $x \in A$. Hence ${ }^{0}(* A) \supset A$.

Definition 3.1. If $\Psi \subset \Gamma, \Psi$ is said to be compact if and only if for each $A \in{ }^{*} \Psi,{ }^{0} A \in \Psi$.

Definition 3.2. Let $\mathcal{C}=\{\Psi \mid \Psi \subset \Gamma$ and $\Psi$ is compact $\}$.

Theorem 3.2. $(\Gamma, \mathcal{C})$ is a topological space.

Proof. (a) $\emptyset, \Gamma \in \mathcal{C}$.

(b) Let $\Delta, \Psi \in \mathcal{C}$ and $A \in \epsilon^{*}(\Delta \cup \Psi)$. Then $A \in\left({ }^{*} \Delta \cup * \Psi\right)$. Thus $A \epsilon^{*} \Delta$ or $A \in \epsilon^{*} \Psi$. Since $\Delta$ and $\Psi$ are compact, this means that ${ }^{0} A \in \Delta$ or ${ }^{0} A \in \Psi$. Therefore, ${ }^{0} A \in(\Delta \cup \Psi)$ and $(\Delta \cup \Psi) \in \mathcal{C}$.

(c) Let $\Delta_{i}, i \in I$, be in $\mathcal{C}$ and $A \in *\left(\bigcap_{i \in I} \Delta_{i}\right)$. Since $*\left(\bigcap_{i \in I} \Delta_{i}\right)=$ $\bigcap_{i \in{ }^{*} I}{ }^{*} \Delta_{i}$ and $I \subset * I, A \in \bigcap_{i \in I}{ }^{*} \Delta_{i}$. Since for each $i \in I, \Delta_{i}$ is compact, it follows that ${ }^{0} A \in \Delta_{i}$. Therefore ${ }^{0} A \in \bigcap_{i \in I} \Delta_{i}$. Thus $\bigcap_{i \in I} \Delta_{i} \in \mathcal{C}$.

Definition 3.2. The topology $\mathcal{C}$ on $\Gamma$ is called the compact topology.

Example 3.1. Let $X$ be the closed unit disk in the Euclidean plane. Let $\Delta$ be the family of all squares that are contained in $X$ and that bave area $\geq 1 / 2$. Then $\Delta$ is a compact family.

Proof. If $A$ is a square and $A \subset C^{*} X$ and area of $A \geq 1 / 2$ then ${ }^{0} A$ is a square and ${ }^{0} A \subset X$ and area of ${ }^{0} A \geq 1 / 2$. (The compactness of $X$ is needed to ensure that ${ }^{0} A$ is a square.)

Example 3.2. Let $X$ be the closed unit disk in the Euclidean plane. Let $D$ be a Jordan curve and $r$ a positive real number. Let $\Delta_{D, r}$ be the family of all Jordan curves $E$ such that $E$ bas area $\geq r, E \subseteq X$, and there is an affine transformation $T$ such that $T(D)=E$. Then $\Delta_{D, r}$ is a compact family. the form

Proof. Let $A \in \epsilon^{*} \Delta_{D, r^{*}}$ Then $A=T(D)$ where $T$ is an affine transformation of

$$
x^{\prime}=a_{1} x+b_{1} y+c_{1}, \quad y^{\prime}=a_{2} x+b_{2} y+c_{2} .
$$

Since each point of $A$ has at most unit distance from the origin, $a_{1}, b_{1}, c_{1}, a_{2}, b_{2}$, $c_{2}$ are all finite numbers. Since $A$ has area $\geq r, a_{1} b_{2}-a_{2} b_{1}$ is not infinitesimal. For each finite (nonstandard) real number $u$, let ${ }^{0} u$ be the standard real number such that ${ }^{0} u \simeq u$. Then ${ }^{0} a_{1},{ }^{0} b_{1},{ }^{0} a_{2},{ }^{0} b_{2},{ }^{0} c_{1}$, and ${ }^{0} c_{2}$ are standard real numbers and ${ }^{0} a_{1}{ }^{0} b_{2}-{ }^{0} a_{2}{ }^{0} b_{1} \neq 0$. Thus if $S$ is the transformation defined by

$$
x^{\prime}={ }^{0} a_{1} x+{ }^{0} b_{1} y+{ }^{0} c_{1}, \quad y^{\prime}={ }^{0} a_{2} x+{ }^{0} b_{2} y+{ }^{0} c_{2}
$$

then $S$ is affine and $S(D)={ }^{0} A$. It is easy to prove that the area of ${ }^{0} A$ is infinitesimally close to area of $A$. This implies that the area of ${ }^{0} A \geq r$. Thus ${ }^{0} A \in$ $\Delta_{D, r^{\circ}}$ 
Example 3.3. Let $X$ be a linear topological space and $\Delta$ the family of all closed convex subsets of $X$. Then $\Delta$ is a compact family. (Note that neither compactness nor boundedness is assumed.)

Proof. Let $A \in \epsilon^{*} \Delta$ and $a, b \in{ }^{0} A$. Then there are $c$ and $d$ in $A$ such that $a \simeq$ $c$ and $b \simeq d$. Since $A$ is convex, for each $t$ (in the enlargement) such that $0 \leq t \leq$ $1,(c t+d(1-t)) \in A$. Therefore, ${ }^{0}(c t+d(1-t)) \in{ }^{0} A$. By an easy argument about near-standardness, it follows that $(a t+b(1-t)) \in{ }^{0} A$ for each standard $t \in[0,1]$. Thus ${ }^{0} A \in \Delta$.

Example 3.4. Let $(X, \Gamma)$ be a topological group and $\Delta$ the family of closed subgroups of $(X, \Gamma)$. Then $\Delta$ is a compact family.

Proof. Let $A \in{ }^{*} \Delta$. We need only to show that ${ }^{0} A \in \Delta$. ${ }^{0} A$ is closed. To show that ${ }^{0} A$ is a group, let $x_{0}, y_{0} \in{ }^{0} A, x, y \in A, x_{0} \simeq x$, and $y_{0} \simeq y$. Then $x_{0} \cdot y_{0} \simeq x \cdot y$. Since $A$ is a group, $x \cdot y \in A$. Thus $x_{0} \cdot y_{0} \in{ }^{0} A$. Since $x_{0}^{-1} \simeq$ $x^{-1}$ and $x^{-1} \in A$, it follows that $x_{0}^{-1} \in{ }^{0} A$. Thus ${ }^{0} A$ is a closed group.

Example 3.5. Mabler families of lattices. Let $R^{n}$ be Euclidean $n$-space. Each $x$ in $R^{n}$ can be represented by an ordered $n$-tuple $\left(x_{1}, \cdots, x_{n}\right)$ of real numbers. Let $\left\{a_{1}, \cdots, a_{n}\right\}$ be a set of linear independent vectors in $R^{n}$ and so $A=$ $\left\{\lambda_{1} a_{1}+\cdots+\lambda_{n} a_{n} \mid \lambda_{1}, \cdots, \lambda_{n}\right.$ are integers $\}$. Then $A$ is said to be an $n$-dimensional lattice generated by $\left\{a_{1}, \cdots, a_{n}\right\}$, and $\left\{a_{1}, \cdots, a_{n}\right\}$ is called a basis for $A$. Since $\left\{a_{1}, \cdots, a_{n}\right\}$ are independent, the determinate $\left|a_{i j}\right|$ is not 0. If $\left\{b_{1}, \cdots, b_{n}\right\}$ is another basis for $A$ then $\left\{b_{1}, \cdots, b_{n}\right\}$ is also independent. Let $d(A)$ be the greatest lower bound of the positive determinates of the form $\left|b_{i j}\right|$, where $\left\{b_{1}, \cdots, b_{n}\right\}$ is a basis for $A$. Let

$$
|A|=\inf _{a \in(A-\{0\})}\|a\|,
$$

where $\|a\|$ is the norm of the vector $a$. A family, $\Delta$, of $n$-dimensional lattices is said to be a Mabler family if and only if for each $A \in \Delta$ there are positive real numbers $r$ and $s$ such that $d(A) \leq r$ and $|A| \geq s$. A. Robinson in [9] shows that if $\Delta$ is a Mahler family and $A \in{ }^{*} \Delta$ then ${ }^{0} A \in \Delta$. Hence a Mabler family is a compact family.

Theorem 3.3. Let $(X, \Gamma)$ be a topological space. $B \epsilon^{*} \Gamma$ can be considered in two ways: as a closed subset of ${ }^{*} X$ and as a point in the space $\left({ }^{*} \Gamma,{ }^{*} \mathcal{C}\right)$. Consider $B$ as a closed subset of ${ }^{*} X$ and let $A={ }^{0} B$. Then in the space $\left({ }^{*} \Gamma,{ }^{*} \mathcal{C}\right), B$ is near-standard to ${ }^{*} A$.

Proof. Assume $B$ is not near-standard to ${ }^{*} A$. Then there is a compact family $\Delta$ in $\mathcal{C}$ such that $B \epsilon^{*} \Delta$ and ${ }^{*} A \epsilon^{*}\left(\Delta^{\sim}\right)$, where $\Delta^{\sim}$ is the complement of $\Delta$. Therefore, ${ }^{*} A \notin^{*} \Delta$. Since $\Delta$ is compact, ${ }^{0} B=A \in \Delta$. Therefore $A \in \Delta$ and ${ }^{*} A \notin^{*} \Delta$. This contradicts elementary equivalence between the standard model and the enlargement. 
Theorem 3.4. $(\Gamma, \mathcal{C})$ is a compact space.

Proof. By The orem 3.3 each point of ${ }^{*} \Gamma$ is near-standard.

The condition "if $A \in{ }^{*} \Delta$ then ${ }^{0} A \in \Delta$ " is satisfied by many important families of closed subsets. It is also an easy condition to verify. This coupled with Theorem 3.4 then gives a powerful method of establishing compactness properties for collections of families of subsets-as the following example will show.

Example 3.6. Let $(X, \Gamma)$ be a compact Hausdorff space. The function $\Pi$ is said to be a dynamical system on $(X, \Gamma)$ if and only if the following two conditions hold:

(1) $\Pi$ is a continuous function from $X \times R$ onto $X$, where $R$ is the real number system,

(2) if $z=\Pi(x, t)$ and $w=\Pi\left(z, t^{\prime}\right)$ then $w=\Pi\left(x, t+t^{\prime}\right)$. Then for each $x \in X$, $\Pi_{x}(t)=\Pi(x, t)$ is a continuous function in the variable $t$, and for each $t \in R$, $\Pi_{t}(x)=\Pi(x, t)$ is a continuous function in the variable $x$. If, in addition, there is a $t_{0} \in R$ such that for each $x \in X$ there is a $t^{\prime} \leq t_{0}$ such that $\Pi_{x}(t)=\Pi_{x}\left(t+t^{\prime}\right)$ for each real number $t, \Pi$ is said to be periodic. The notion of orbit is one of the fundamental concepts in the theory of dynamical systems. For each $x \in X, A_{x}=$ $\left\{y \mid y=\Pi_{x}(t)\right.$ for some $\left.t \in R\right\}$ is called the orbit of $x$ under $\Pi$. Since $y \in A_{x}$ if and only if $x \in A_{y}, X$ is partitioned by the family of orbits that are determined by a given dynamical system on $(X, \Gamma)$. If $\mathcal{F}$ is a family of dynamical systems on the space $(X, \Gamma), \mathcal{F}$ is said to have a fixed orbit if and only if there is a set $A$ such that for each $\Pi$ in $\mathcal{F}$, there is an $x$ in $X$ such that $A$ is the orbit of $x$ under $\Pi$. The following compactness theorem can now be proved:

If $(X, \Gamma)$ is a compact Hausdorff space, $\mathcal{F}$ is a family of periodic dynamical systems on $(X, \Gamma)$, and eacb finite subfamily of $\mathcal{F}$ bas a fixed orbit, then $\mathcal{F}$ bas a fixed orbit.

Proof. Let $\Pi \in \mathcal{F}, \Delta$ the family of orbits determined by $\Pi$, and $A \in \Delta$. Then for some $x$ in $X, A=\left\{\Pi_{x}(t) \mid t \in R\right\}$. Since $\Pi$ is periodic, there are $t_{1}$ and $t_{2}$ such that $A=\left\{\Pi_{x}(t) \mid t \in\left[t_{1}, t_{2}\right]\right\}$. Since $A$ is the image of a compact set under a continuous function, $A$ is compact and therefore closed. Thus $\Delta \subset \Gamma$. Let $D \in{ }^{*} \Delta$, $x \in{ }^{*} X$, and $D=\left\{{ }^{*} \Pi_{x}(t) \mid t \in{ }^{*} R\right\}$. Since $X$ is a compact Hausdorff space, let $x_{0}$ be the unique member of $X$ such that $x_{0} \simeq x$. Let $B$ be the orbit of $x_{0}$ under $\Pi$. Then $B={ }^{0} D$. For if $z \in B$, then there is a $t$ in $R$ such that $z=\Pi\left(x_{0}, t\right)=\Pi_{t}\left(x_{0}\right)$. Since $\Pi_{t}$ is a standard continuous function, $z={ }^{*} \Pi_{t}\left(x_{0}\right) \simeq{ }^{*} \Pi_{t}(x)={ }^{*} \Pi_{x}(t) \in D$. Thus $B \subset{ }^{0} D$. To show ${ }^{0} D \subset B$, let $z \in{ }^{0} D$. Then $z \simeq \simeq^{*} \Pi(x, u)$ for some $u \in \epsilon^{*} R$. Since $\Pi$ is periodic we may suppose that $u$ is finite. Let $u_{0}$ be the real number that is near-standard to $u$. Since $(X, \Gamma)$ is Hausdorff, $z=\Pi\left(x_{0}, u_{0}\right)$. Thus $z \in B$ and ${ }^{0} D \subset B$. Since $B={ }^{0} D,{ }^{0} D \in \Delta$. Hence $\Delta$ is a compact family. For each $\Pi$ in $\mathcal{F}$ let $\Delta(\Pi)$ be the family of orbits determined by $\Pi$. If $\mathcal{G} \subset \mathcal{F}$ then $\mathcal{G}$ has a fixed 
orbit if and only if $\bigcap_{\Pi \in \mathcal{S}} \Delta(\Pi) \neq \varnothing$. Since each finite subfamily $\mathcal{G}$ of $\mathcal{F}$ has a fixed orbit and $\Delta(I I) \in \mathcal{C}$ for each $\Pi$ in $\mathcal{F}$, it then follows from the compactness of $\left(\Gamma, \mathcal{C}\right.$ ) (Theorem 3.4) that $\bigcap_{\Pi \in \mathcal{G}} \Delta(\Pi) \neq \varnothing$.

Example 3.7. Let $(X, \Gamma)$ be the discrete space. That is, let $\Gamma$ be the family of all subsets of $X$. Then $(\Gamma, \mathcal{C})$ is a compact Hausdorff space.

Proof. By Theorem 3.4, $(\Gamma, \mathcal{C})$ is compact. To see that $(\Gamma, \mathcal{C})$ is also a Hausdorff space, let $A, B \in \Gamma$ and $A \neq B$. Without loss of generality we may let $a$ be a point of $X$ such that $a \in A$ and $a \notin B$. Let $\Delta=\{D \in \Gamma \mid a \in D\}$ and $\Psi=$ $\{D \in \Gamma \mid a \notin D\}$. Then $\Delta$ and $\Psi$ are in $\mathcal{C}$. Thus in the space $(\Gamma, \mathcal{C}), \Delta^{\sim}$ and $\Psi^{\sim}$ are open sets with $B \in \Delta^{\sim}, A \in \Psi^{\sim}$, and $\Delta^{\sim} \cap \Psi^{\sim}=\emptyset$.

Some closed families of the discrete space are: $\{A \mid A \subset X$ and $A$ has less than $n$ members $\}$ where $n$ is a positive integer, and having less then $n$ members allows the case of having no members; $\{A \mid D \subset A\}$ and $\{A \mid A \subset D\}$ where $D$ is a subset of $X$. Observe that $\{A \mid A \subset X$ and $A$ has exactly 2 members $\}$ may not be closed in the compact topology.

Definition 3.3. A point $x$ in the topological space $X$ is said to be isolated if and only if $X-\{x\}$ is a closed subset of $X$.

Theorem 3.5. $(X, \Gamma)$ is compact if and only if $\emptyset$ is an isolated point of the space $(\Gamma, \mathcal{C})$.

Proof. Suppose $(X, \Gamma)$ is compact. Let $\Delta=\{A \mid A \in \Gamma$ and $A \neq \emptyset\}$. If $A \epsilon^{*} \Delta$ then ${ }^{0} A$ is closed. Since $(X, \Gamma)$ is compact and $A \neq \emptyset,{ }^{0} A$ is nonempty. Thus ${ }^{0} A \in \Delta$. Therefore $\Delta$ is a closed subset of $(\Gamma, \mathcal{C})$. Since $\Delta=\Gamma-\{\varnothing\}, \emptyset$ is isolated.

Suppose that $\emptyset$ is an isolated point of $(\Gamma, \mathcal{C})$. Then $\Delta=\{A \mid A \in \Gamma$ and $A \neq \emptyset\}$ is a closed subset of $\Gamma$. Let $x \epsilon^{*} X$. It will be shown that $x$ is near-standard. In the enlargement, let $\Psi=\left\{A \mid A \epsilon^{*} \Gamma\right.$ and $\left.x \in A\right\}$ and $B=\bigcap \Psi$. Then $B \epsilon^{*} \Delta$. Since $\Delta \in \mathcal{C},{ }^{0} B \in \Delta$. Therefore ${ }^{0} B \neq \emptyset$. Let $y \in{ }^{0} B$ and $U$ an arbitrary open set that contains $y$. We will show that $x \in \epsilon^{*} U$. For suppose $x \notin^{*} U$. Then $x \in \epsilon^{*} U^{\sim}$ and ${ }^{*} U^{\sim} \epsilon{ }^{*} \Gamma$. By the definition of $B, B \subset^{*} U^{\sim}$. However, since $y \in{ }^{0} B,{ }^{*} U \cap B \neq \varnothing-$ a contradiction. Therefore $x \in{ }^{*} U$. Since $U$ is an arbitrary open set containing $y$, it follows that $x$ is in the monad of $y$. Thus $(X, \Gamma)$ is a compact space.

Theorem 3.6. If $(X, \Gamma)$ is a locally compact $T_{3}$ space then $(\Gamma, \mathcal{C})$ is a Hausdorff space.

Proof. Let $A, B \in \Gamma$ and $A \neq B$. Without loss of generality, assume that $a \in A$ and $a \notin B$. Since $(X, \Gamma)$ is a locally compact $T_{3}$ space, let $U$ and $V$ be open subsets of $X$ such that $a \in U, B \subset V, U \cap V=\varnothing$, and $U^{c}$, the closure of $U$, is compact. $U^{c} \cap V=\varnothing$. For if $x \in U^{c} \cap V$ then, since $V$ is an open subset of $X, V \cap$ $U \neq \emptyset$-a contradiction. In particular, $U^{c} \cap B=\emptyset$. Let $\Delta=\left\{D \in \Gamma \mid D \subset U^{\sim}\right\}$ and 
$\Psi=\left\{D \in \Gamma \mid D \cap U^{c} \neq \emptyset\right\} . \Delta \in \mathcal{C}$. Since $U^{c}$ is compact, $\Psi \in \mathcal{C}$. Since $A \in \Delta^{\sim}$, $B \in \Psi \sim$, and $\Delta^{\sim} \cap \Psi^{\sim}=\{D \in \Gamma \mid D \cap U \neq \emptyset\} \cap\left\{D \in \Gamma \mid D \cap U^{c}=\emptyset\right\}=\emptyset$, it follows that $(\Gamma, \mathcal{C})$ is a Hausdorff space.

Definition 3.5. Let $(X, \Gamma)$ be a topological space. For each finite family of open subsets of $X, G_{0}, G_{1}, \cdots, G_{n}(n \geq 0)$, let

$$
\Re\left(G_{0}, G_{1}, \cdots, G_{n}\right)=\left\{A \in \Gamma \mid A \subset G_{0} \text { and for } i=1, \cdots, n, G_{i} \cap A \neq \emptyset\right\} .
$$

The Vietoris topology on $\Gamma$ is the topology on $\Gamma$ which has families of the form $\mathcal{B}\left(G_{0}, G_{1}, \cdots, G_{n}\right)$ as basic open sets, where $G_{0}, \cdots, G_{n}$ are an arbitrary, finite number of open subsets of $X$. (See [3, pp. 160-169] and [11].) Let $\mathcal{O}$ denote the collection of closed families of the Vietoris topology.

Theorem 3.7. If $(X, \Gamma)$ is compact then $\mathcal{O} \subset \mathcal{U}$.

Proof. Suppose $\triangle \in \mathcal{O}$ and $A \in{ }^{*} \Delta$. Let $B\left(G_{0}, \cdots, G_{n}\right)$ be an arbitrary, basic open set in the Vietoris topology such that ${ }^{0} A \in \mathcal{B}\left(G_{0}, \cdots, G_{n}\right)$. That is, ${ }^{0} A \subset$ $G_{0}$ and for $i=1, \cdots, n, G_{i} \cap{ }^{0} A \neq \emptyset$. Let $1 \leq i \leq n$ and $x_{i} \in G_{i} \cap{ }^{0} A$. By the definition of monad, in the enlargement let $y_{i}$ be such that $y_{i} \in A$ and $x_{i} \simeq y_{i}$. By the definition of monad, $y_{i} \in{ }^{*} G_{i}$. Hence for $i=1, \cdots, n,{ }^{*} G_{i} \cap A \neq \emptyset$. Let $y \in A$. Since $(X, \Gamma)$ is compact, let $x \in X$ and such that $x \simeq y$. Since $x \in{ }^{0} A, x \in G_{0}$. Thus by the definition of monad, $y \in{ }^{*} G_{0}$. Hence $A \subset{ }^{*} G_{0}$. Thus $A \epsilon$ ${ }^{*} B\left({ }^{*} G_{0}, \cdots,{ }^{*} G_{n}\right)$. Therefore in the enlargement, ${ }^{*} \Delta \cap{ }^{*} \mathfrak{B}\left({ }^{*} G_{0}, \cdots,{ }^{*} G_{n}\right) \neq \varnothing$. Hence $\Delta \cap \mathfrak{B}\left(G_{0}, \cdots, G_{n}\right) \neq \varnothing$. Thus in the Vietoris topology, ${ }^{0} A$ is in the closure of $\Delta$. Since $\Delta$ is closed in the Vietoris topology, ${ }^{0} A \in \Delta$. Therefore $\Delta \in \mathcal{C}$.

Theorem 3.8. If $(X, \Gamma)$ is a compact Hausdorff space then $\mathcal{O}=\mathcal{C}$.

Proof. By Theorem 3.7, $0 \subset \mathcal{C}$. Let $\Psi \in \mathcal{C}$. Let $E$ be an accumulation point of $\Psi$ in the Vietoris topology. It will be shown that $E \in \Psi$. Let $R(U, \mathcal{F})$ be the following relation:

$$
\begin{aligned}
& \text { " } U \text { is an open subset of } X, \mathcal{F} \text { is a finite family of subsets, } \\
& U \in \mathcal{F} \text {, and for each } V \in \mathcal{F}, V \text { is an open subset of } X \text { and } \\
& V \cap E \neq \emptyset . "
\end{aligned}
$$

Then $R(U, \mathcal{F})$ is a concurrent relation: for if $R\left(U_{1}, \mathcal{F}_{1}\right), \cdots, R\left(U_{n}, \mathcal{F}_{n}\right)$ hold in the standard model, then $R\left(U_{1}, \mathcal{F}\right), \cdots, R\left(U_{n}, \mathcal{F}\right)$ also hold in the standard model, where $\mathcal{F}=\bigcup_{i=1}^{n} \mathfrak{F}_{i}$. Therefore, let $\mathcal{G}$ be an element of the enlargement such that for all standard $U$ in the domain of $R, \mathbf{R}\left({ }^{*} U, \mathcal{G}\right)$ is true in the enlargement. Since $\mathcal{G}$ is (nonstandardly) finite, let $\oint_{1}=\left\{G_{1}, G_{2}, \cdots, G_{p}\right\}$, where $p$ is a (nonstandard) natural number. Let $S(U, G)$ be the following concurrent relation:

$$
\text { " } G \text { and } U \text { are open subsets of } X \text { and } E \subset G \subset U \text {." }
$$

In the enlargement, let $G_{0}$ be such that for all standard $U$ in the domain of $S$, $\mathrm{S}\left({ }^{*} U, G_{0}\right)$. Then ${ }^{*} E \in \mathfrak{B}\left(G_{0}, G_{1}, \cdots, G_{p}\right)$. Since ${ }^{*} E$ is an accumulation point of 
${ }^{*} \Psi$ in the ${ }^{*}$ Vietoris topology, $\mathbb{B}\left(G_{0}, G_{1}, \cdots, G_{p}\right) \cap{ }^{*} \Psi \neq \varnothing$. Let $D \in$ $\Re\left(G_{0}, G_{1}, \cdots, G_{p}\right) \cap{ }^{*} \Psi$. We will show that ${ }^{0} D=E$. Let $x \in{ }^{0} D$. Assume $x \notin E$. A contradiction will be shown. Since $(X, \Gamma)$ is a compact Hausdorff space, there are open subsets of $X, U_{1}$ and $U_{2}$, such that $x \in U_{1}, E \subset U_{2}$, and $U_{1} \cap U_{2}=\varnothing$. Since $G_{0} \subset{ }^{*} U_{2},{ }^{*} U_{1} \cap G_{0}=\emptyset$. Since $D \subset G_{0},{ }^{*} U_{1} \cap D=\emptyset$. However, since $x \in$ ${ }^{0} D$, there is a $y \in D$ such that $x \simeq y$. By the definition of monad, $y \in{ }^{*} U_{1}$. Hence ${ }^{*} U_{1} \cap D \neq \emptyset$. A contradiction. Thus $x \in E$. Therefore ${ }^{0} D \subset E$. Let $z \in E$. Suppose $z \notin^{0} D$. A contradiction will be shown. Since $(X, \Gamma)$ is a compact Hausdorff space, there are open subsets $V$ and $W$ of $X$ such that $x \in V,{ }^{0} D \subset W$, and $V \cap W=$ $\emptyset$. Since ${ }^{*} V \in \mathcal{G},{ }^{*} V \cap D \neq \varnothing$. Let $v \in^{*} V \cap D$. By the compactness of $(X, \Gamma)$, let $u$ be a standard element such that $u \simeq v$. Then $u \in{ }^{0} D$. Since $W$ is an open set containing $u$ and $u \simeq v$, by the definition of monad, $v \in{ }^{*} W$. Hence ${ }^{*} V \cap{ }^{*} W \neq$ $\emptyset$. Therefore, $V \cap W \neq \emptyset$, a contradiction. Hence $E \subset{ }^{0} D$. Thus we have shown $E={ }^{0} D$. Since $\Psi \in \mathcal{C}$ and $D \in{ }^{*} \Psi,{ }^{0} D=E \in \Psi$. Thus $\Psi \in \mathcal{O}$. Therefore $\mathcal{C} \subset \mathcal{O}$.

Theorem 3.9. If $(X, \Gamma)$ is not a compact space then $\mathcal{C} \neq \mathcal{C}$.

Proof. Since $B(\emptyset)=\{\varnothing\},\{\varnothing\}$ is an open set in the Vietoris topology. By Theorem 3.5, $\{\varnothing\}$ is not an open set in the compact topology.

The following example will more clearly show the differences of the Vietoris topology and the compact topology.

Example 3.8. Let $(X, \Gamma)$ be the Euclidean plane. Let $\Delta$ be the family of all $A_{n}$, where $n$ is a natural number, such that $A_{n}$ is the union of two perpendicular lines, one of which is the $Y$-axis, and the other a line through $(0, n)$. Let $D$ be an accumulation point of $\Delta$ in the Vietoris topology. We first note that $D$ is not a subset of the $Y$-axis: for if $D$ were a subset of the $Y$-axis, then for $V=\{(x, y) \mid-1 / 2<$ $x<1 / 2\}, D \in \mathcal{B}(V)$, and for each $A \in \Delta, A \notin \mathfrak{B}(V)$, thus contradicting that $D$ is an accumulation point of $\Delta$. Next we note that if $b \in D \cap(Y$-axis) $\sim$, and $U$ is the open disk about $b$ of radius $1 / 2, D \in \mathfrak{B}(X, U)$, and since $U$ intersects at most one member of $\Delta$, at most one member of $\Delta$ is in $B(X, U)$, thus making it impossible for $D$ to be an accumulation point of $\Delta$. Thus $\Delta$ contains all of its accumulation points in the Vietoris topology. Therefore $\Delta \in \mathcal{O}$. Let $p$ be an infinite natural, number. Then $A_{p} \in{ }^{*} \Delta$ but the $Y$-axis $={ }^{0} A_{p} \notin \Delta$. Hence $\Delta \notin \mathcal{C}$. Observe that if $\Psi=\Delta \cup\{Y$ axis $\}$ then $\Psi$ is a compact family.

\section{Metric spaces.}

Definition 4.1. Let $(X, \Gamma)$ be a metric space with metric $\rho$. If $x$ is in $X$ and $A$ is in $\Gamma$ then the distance from $x$ to $A, \rho(x, A)$, is defined as follows:

$$
\rho(x, A)=\inf _{y \in A} \rho(x, y) \text {. }
$$

The diameter of $X, \delta(X)$, is defined as follows:

$$
\delta(X)=\sup _{x, y \leq X} \rho(x, y) .
$$


$(X, \Gamma)$ is said to be bounded if and only if the diameter of $X$ is finite. If $A, B \in \Gamma$ then the distance from $A$ to $B, \rho(A, B)$, is defined as follows:

(1) if $A \neq \emptyset$ and $B \neq \varnothing$ let $a=\sup _{x \in A} \rho(x, B)$ and $b=\sup _{x \in B} \rho(x, A)$ and $\rho(A, B)=\sup \{a, b\}$;

(2) if $A=\emptyset$ and $B \neq \emptyset$ let $\rho(A, B)=\rho(B, A)=\delta(X)+1$;

(3) if $A=B=\varnothing$ let $\rho(A, B)=0$.

Theorem 4.1. If $(X, \Gamma)$ is a metric space with metric $\rho$ and $A, B, D \in \Gamma$ then the following statements are true:

(1) $\rho(A, B) \in[0, \infty]$ ( $\infty$ can only be assumed in unbounded spaces),

(2) $\rho(A, B)=\rho(B, A)$,

(3) $\rho(A, B) \leq \rho(A, D)+\rho(B, D)$,

(4) $\rho(A, B)=0$ if and only if $A=B$,

(5) if $A \neq \emptyset$ then $\rho(A, \emptyset)=$ diameter of $X$.

The proof of this theorem is left to the reader.

Definition 4.2. If $(X, \Gamma)$ is a bounded metric space with metric $\rho$ then, by Theorem 4.1, $\rho$ defines a metric on $\Gamma$. This metric is called the Hausdorff metric on $\Gamma$. Let $\mathcal{H}=\{\Delta \mid \Delta$ is a closed subset of $\Gamma$ in the topology determined by the Hausdorff metric\}. $\mathcal{H}$ is called the Hausdorff topology on $\Gamma$.

Theorem 4.2. If $(X, \Gamma)$ is a bounded metric space then $(\Gamma, \mathcal{H})$ is a bounded metric space.

Proof. Immediate from Definition 4.2.

Theorem 4.3. If $(X, \Gamma)$ is a bounded metric space, $\mathcal{C}$ is the compact topology on $\Gamma$, and $\mathcal{H}$ the Hausdorff topology on $\Gamma$, then $\mathcal{C} \subset \mathcal{H}$.

Proof. Suppose $(X, \Gamma)$ is a bounded metric space with metric $\rho, \Delta \in \mathcal{C}$, and in the Hausdorff topology, $A$ is an accumulation point of $\Delta$. That is, for each positive real number $r$, there is a $B$ in $\Delta$ such that $A \neq B$ and $\rho(A, B)<r$. Therefore, in the enlargement, there is a $B$ in ${ }^{*} \Delta$ such that ${ }^{*} A \neq B$ and ${ }^{*} \rho\left({ }^{*} A, B\right) \simeq 0$. But ${ }^{*}\left({ }^{*} A, B\right) \simeq 0$ if and only if for each $x \in{ }^{*} A$ there is a $y \in B$ such that ${ }^{*} \rho(x, y) \simeq 0$ and for each $v \in B$ there is a $z \in{ }^{*} A$ such that ${ }^{*} \rho(v, z) \simeq 0$. Thus ${ }^{0}\left({ }^{*} A\right)={ }^{0} B$. Since ${ }^{0}\left({ }^{*} A\right)=A$ (Theorem 3.1), $A={ }^{0} B$. Since $\Delta$ is in $\mathcal{C}$ and ${ }^{*} B$ is in ${ }^{*} \Delta,{ }^{0} B=$ $A \in \Delta$. Thus $\Delta$ contains (in the topology $\mathcal{H}$ ) all of its accumulation points. Therefore $\Delta \in \mathcal{H}$.

Theorem 4.4. If $(X, \Gamma)$ is a compact metric space then $\mathcal{C}=\mathcal{H}$.

Proof. Assume $(X, \Gamma)$ is a compact metric space with metric $\rho$. By Theorem 4.3, $\mathcal{C} \subset \mathcal{H}$. Let $\Delta \in \mathcal{H}$. Suppose that in the enlargement $A \in \epsilon^{*} \Delta$, and in the standard model, ${ }^{0} A \notin \Delta$. A contradiction will be shown. Since ${ }^{0} A \notin \Delta$ and $\Delta \in \mathcal{H}$, there is a standard positive $r$ such that for each $B \in \Delta, \rho\left({ }^{0} A, B\right)>r$. Therefore, 
in the enlargement, ${ }^{*} \rho\left({ }^{*}\left({ }^{0} A\right), A\right)>r$. Since $X$ is compact, if $y \in A$ there is an $x \in{ }^{0} A$ such that ${ }^{*} \rho(x, y) \simeq 0$. Therefore, for each $y \in A,{ }^{*} \rho\left(y,{ }^{*}\left({ }^{0} A\right)\right) \simeq 0$. Hence, since ${ }^{*}\left({ }^{*}\left({ }^{0} A\right), A\right)>r$, there is a $z \in{ }^{*}\left({ }^{0} A\right)$ such that ${ }^{*} \rho(z, A)>r$. Since $(X, \Gamma)$ is compact, there is a $v \in X$ such that $v \simeq z$. Thus ${ }^{*} \rho(v, A)>r$. Hence $v \notin^{0} A$. But, by Theorem 3.1, ${ }^{0}\left({ }^{*}\left({ }^{0} A\right)\right)={ }^{0} A$, and since $z \in{ }^{*}\left({ }^{0} A\right)$ and $v \simeq z, v \in{ }^{0}\left({ }^{*}\left({ }^{0} A\right)\right)=$ ${ }^{0} A$. A contradiction. Therefore we can conclude that $\mathcal{H} \subset \mathcal{C}$ and thus $\mathcal{H}=\mathcal{C}$.

Corollary 4.1 (Vietoris). If $(X, \Gamma)$ is a compact metric space then $(\Gamma, \mathcal{H})$ is a compact metric space.

Definition 4.3. Let $(X, \Gamma)$ be a metric space with metric $\rho$. If for each natural number $n, A_{n} \in \Gamma$, then $\left\{A_{n} \mid n\right.$ is a natural number $\}$ is said to be a Cauchy sequence if and only if for each real number $r>0$, there is a natural number $n$ such that for all natural numbers $m$ and $p$, if $m, p>n$ then $\rho\left(A_{m}, A_{p}\right)<r$.

Definition 4.4. Let $(X, \Gamma)$ be a metric space with metric $\rho$. If $A_{n} \in \Gamma$ for each natural number, then $A=\operatorname{Lim} A_{n}$ if and only if $\lim _{n \rightarrow \infty} \rho\left(A, A_{n}\right)=0$.

Example 4.1. Let $(X, \Gamma)$ be the Euclidean plane, $x_{n}$ the point $(n, 0)$, and $A_{n}=\left\{x_{n}\right\}$. Then $\operatorname{Lim} A_{n}$ does not exist. That is, there is no $A$ such that $A=$ $\operatorname{Lim} A_{n^{*}}$ In particular, $\emptyset \neq \operatorname{Lim} A_{n}$.

Theorem 4.5. Let $(X, \Gamma)$ be a metric space, $\left\{A_{n}\right\}$ a sequence of closed subsets of $X$, and $A=\operatorname{Lim} A_{n}$. Then for each infinite natural number $p, A={ }^{0} A_{p}$.

Proof. Let $\rho$ be a metric for $(X, \Gamma)$. If $r$ is an arbitrary, standard, positive, real number, then from Definition 4.4 it follows that ${ }^{*} \rho\left({ }^{*} A,{ }^{*} A_{p}\right)<r$. Hence ${ }^{*} \rho\left({ }^{*} A,{ }^{*} A_{p}\right) \simeq 0$. Therefore, $\rho\left({ }^{0}\left({ }^{*} A\right),{ }^{0} A_{p}\right)=0$. Since ${ }^{0}\left({ }^{*} A\right)=A, \rho\left(A,{ }^{0} A_{p}\right)=0$. That is, $A={ }^{0} A_{p}$.

Definition 4.5. Let $(X, \Gamma)$ be a metric space with metric $\rho$. Then $(X, \Gamma)$ is said to be uniformly locally compact if and only if for each $r>0$ and each $x \in X$, $\{y \in X \mid \rho(x, y) \leq r\}$ is compact.

Theorem 4.6. Let $(X, \Gamma)$ be a uniformly locally compact metric space and $\left\{A_{n}\right\}$ be a Cauchy sequence of closed subsets of $X$. Then $\operatorname{Lim} A_{n}$ exists. In fact, for each infinite natural number $p, \operatorname{Lim} A_{n}={ }^{0} A_{p}$.

Proof. Let $\rho$ be a metric for $(X, \Gamma)$. In the enlargement, let $p$ and $q$ be two infinite natural numbers. It then follows from the definition of Cauchy sequence that ${ }^{*} \rho\left(A_{p}, A_{q}\right) \simeq 0$. Hence ${ }^{0} A_{p}={ }^{0} A_{q}$. Let $A={ }^{0} A_{p}$ and suppose that $A \neq$ $\operatorname{Lim} A_{n}$. A contradiction will be shown. Since $A \neq \operatorname{Lim} A_{n}$, it is not the case that $\lim _{n \rightarrow \infty} \rho\left(A, A_{n}\right)=0$. Hence there is a positive real number $r$ such that $\rho\left(A, A_{n}\right) \geq$ $r$ for infinitely many natural numbers $n$. Since each subsequence of a Cauchy sequence is a Cauchy sequence, we may suppose, without loss of generality, that $\left\{A_{n}\right\}$ is a Cauchy sequence and $\rho\left(A, A_{n}\right) \geq r$ for each natural number $n$. Then at 
least one of the following two cases holds:

(1) for some $x$ in $A, \rho\left(x, A_{n}\right) \geq r$ for infinitely many $n$,

(2) there is an $m_{0}$ such that for all $n>m_{0}$, there is an $x_{n} \in A_{n}$ such that $\rho\left(x_{n}, A\right) \geq r$.

Assume case (1). Then for some infinite natural number $t,{ }^{*} \rho\left(x, A_{t}\right) \geq r$. But since ${ }^{0} A_{t}=A,{ }^{*} \rho\left(x, A_{t}\right) \simeq 0$. A contradiction. Case (2) is also impossible. For let $m$ be a natural number such that $m>m_{0}$ and for all $s, n \geq m, \rho\left(A_{s}, A_{n}\right)<1 / 2 r$. Then for some infinite natural number $t,{ }^{*} \rho\left({ }^{*} A_{m}, A_{t}\right)<1 / 2 r$. In particular, ${ }^{*} \rho\left(x_{m}, A_{t}\right)$ $<1 / 2 r$. Let $\alpha \in A_{t}$ and be such that ${ }^{*} \rho\left(x_{m}, a\right) \leq 1 / 2 r$. By hypothesis, $B=\{z \epsilon$ $\left.X \mid \rho\left(x_{m}, z\right) \leq 1 / 2 r\right\}$ is a compact set. Since $A \epsilon^{*} B$, let $a_{0}$ be a standard point in $B$ such that $a_{0} \simeq a$. Then $a_{0} \in{ }^{0} A_{t}=A$. Therefore, $\rho\left(x_{m}, A\right) \leq r / 2$. A contradiction.

Definition 4.6. Let $(X, \Gamma)$ be a metric space and $\Delta C \Gamma . \Delta$ is said to be complete if and only if whenever $\left\{A_{n}\right\}$ is a Cauchy sequence such that for each $n$, $A_{n} \in \Delta, \operatorname{Lim} A_{n}$ exists and $\operatorname{Lim} A_{n} \in \Delta$.

Note that the definition of completeness for families of closed subsets does not assume that the underlying space, $(X, \Gamma)$, is bounded. Since the Hausdorff topology is only defined for bounded spaces, the above notion of completeness applies to some nonmetric spaces.

Theorem 4.7. If $(X, \Gamma)$ is a uniformly locally compact metric space and $\Delta \in \mathcal{C}$ then $\Delta$ is a complete family.

Proof. Suppose that $(X, \Gamma)$ is a uniformly locally compact metric space with metric $\rho$ and $\Delta \in \mathcal{C}$. Also suppose that $\left\{A_{n}\right\}$ is a Cauchy sequence such that for each natural number $n, A_{n} \in \Delta$. In the enlargement, let $p$ be an infinite natural number. Then $A_{p} \in{ }^{*} \Delta$. Let $A={ }^{0} A_{p}$. By Theorem 4.6, $A=\operatorname{Lim} A_{n}$. Since $\Delta \epsilon$ $\mathcal{C}, A={ }^{0} A_{p} \in \Delta$. Thus $\Delta$ is complete.

Example 4.2. An example of a compact family that is not a complete family. Let $(X, \Gamma)$ be the rational numbers with the usual metric $\rho(x, y)=|x-y|$. Let $\Delta=\{\{x\} \mid x \in X\} \cup\{\varnothing\}$. Then $\Delta$ is a compact family. Let $\left\{a_{n}\right\}$ be a sequence of rational numbers that converges to $\sqrt{2}$. Let $A_{n}=\left\{a_{n}\right\}$. Then for each natural number $n, A_{n} \in \Delta .\left\{A_{n}\right\}$ is a Cauchy sequence, but $\operatorname{Lim} A_{n}$ does not exist. Therefore $\Delta$ is not a complete family.

Example 4.3. An example of a complete family of closed subsets of the Euclidean plane that is not a compact family. Let $(X, \Gamma)$ be the Euclidean plane $w$ ith its usual metric, $\rho$. Let $\Delta$ be the set of all lines. To show that $\Delta$ is a complete family, let $\left\{A_{n}\right\}$ be a Cauchy sequence such that for each natural number $n$, $A_{n} \in \Delta$. Let $m$ be a natural number such that for all natural numbers $n, q \geq m$, $\rho\left(A_{n}, A_{q}\right)<1$. Thus if $n>m, A_{n}$ is parallel to $A_{m}$. (Otherwise, $\rho\left(A_{m}, A_{n}\right)=\infty$.) In the enlargement, let $\rho$ be an infinite natural number. Then ${ }^{*} \rho\left({ }^{*} A_{m}, A_{p}\right)<1$. 
Hence $A_{p}$ is a line contained in ${ }^{*} X$ that is parallel to ${ }^{*} A_{m}$. Since ${ }^{*} \rho\left({ }^{*} A_{m}, A_{p}\right)<$ $1,{ }^{0} A_{p}$ is a line parallel to $A_{m}$. Hence ${ }^{0} A_{p} \in \Delta$. By Theorem 4.6, $\operatorname{Lim} A_{n}$ exists, and, by Theorem $4.5, \operatorname{Lim} A_{n}={ }^{0} A_{p}$. Therefore $\Delta$ is complete. $\Delta$ is not compact; since if $q$ is an infinite natural number and $A$ is the line through $(q, 0)$ parallel to the $Y$-axis, then $A \in{ }^{*} \Delta$ but ${ }^{0} A=\emptyset \notin \Delta$.

Theorem 4.8. Let $(X, \Gamma)$ be a compact metric space and $\Delta \subset \Gamma$. Then $\Delta$ is a compact family if and only if $\Delta$ is a complete family.

Proof. Assume $\Delta$ is a compact family. Then, by Theorem $4.7, \Delta$ is a complete family. Assume that $\Delta$ is a complete family. Let $\rho$ be a metric for $(X, \Gamma)$. Let $A \in{ }^{*} \Delta$. We will first show that ${ }^{*} \rho\left(A,{ }^{*}\left({ }^{0} A\right)\right) \simeq 0$. Suppose that there is a standard $r>0$ such that ${ }^{*} \rho\left(A,{ }^{*}\left({ }^{0} A\right)\right) \geq r$. Then, since $(X, \Gamma)$ is compact, $\rho\left({ }^{0} A,{ }^{0}\left({ }^{*}\left({ }^{0} A\right)\right)\right) \geq r$. By Theorem 3.1, ${ }^{0}\left({ }^{*}\left({ }^{0} A\right)\right)={ }^{0} A$. Hence $\rho\left({ }^{0} A,{ }^{0} A\right) \geq r$, which is impossible. Thus ${ }^{*} \rho\left(A,{ }^{*}\left({ }^{0} A\right)\right) \simeq 0$. Let $\Delta_{n}=\left\{B \in \Delta \mid \rho\left(B,{ }^{0} A\right) \leq 1 / n\right\}$. Since $A \epsilon^{*} \Delta_{n}$ for each standard natural number $n$, the sentence " ${ }^{*} \Delta_{n} \neq \varnothing$ " is true in the enlargement for each standard natural number $n$. By elementary equivalence, " $\Delta_{n} \neq \varnothing$ " is true in the standard model for all natural numbers $n$. By the axiom of choice, choose $A_{n} \in \Delta_{n}$. Then $\left\{A_{n}\right\}$ is a sequence of members of $\Delta$ and ${ }^{0} A=$ $\operatorname{Lim} A_{n}$. Since every convergent sequence is a Cauchy sequence, $\left\{A_{n}\right\}$ is a Cauchy sequence. Since $\Delta$ is complete, $\operatorname{Lim} A_{n}={ }^{0} A \in \Delta$. Thus $\Delta$ is compact.

\section{Topological limits.}

Definition 5.1. Let $(X, \Gamma)$ be a topological space and $\left\{A_{i}\right\}$ be a sequence of closed subsets of $X$. Then $\liminf \left\{A_{i}\right\}, \lim \sup \left\{A_{i}\right\}$, and $\lim A_{i}$ are defined as follows:

(1) $x \in \liminf \left\{A_{i}\right\}$ if and only if for each open neighborhood $U$ of $x, U \cap A_{i} \neq$ $\emptyset$ for all but finitely many natural numbers $i$,

(2) $x \in \lim \sup \left\{A_{i}\right\}$ if and only if for each neighborhood $U$ of $x, U \cap A_{i} \neq \varnothing$ for infinitely many natural numbers $i$, and

(3) if $\lim \inf \left\{A_{i}\right\}=\lim \sup \left\{A_{i}\right\}=A$ then we say that $A=\lim A_{i}$.

$\lim A_{i}$ is called the topological limit of the sequence $\left\{A_{i}\right\}$.

Theorem 5.1. If $(X, \Gamma)$ is a topological space and $\left\{A_{i}\right\}$ is a sequence of members of $\Gamma$ then $\liminf A_{i}, \limsup A_{i}$, and $\lim A_{i}$, if it exists, are members of $\Gamma$.

The proof is left to the reader.

Definition 5.2. Let $(X, \Gamma)$ be a topological space and $\Delta \subset \Gamma . \Delta$ is said to be a limit family if and only if for each sequence of closed subsets of $X,\left\{A_{i}\right\}$, if $A_{i} \in \Delta$ for each $i$ and $A=\lim A_{i}$ then $A \in \Delta$. $\mathscr{P}$ will denote the set of limit families.

Theorem 5.2. Let $(X, \Gamma)$ be a topological space. Then $(\Gamma, £)$ is a topological space. 
Proof. By Definition 5.2, $\Gamma \in \mathcal{L}$ and $\emptyset \in \mathfrak{L}$. Suppose that $\Delta$ and $\Psi$ are in $\mathscr{L}$. It will be shown that $\Delta \cup \Psi$ is in $\mathcal{\varrho}$. Suppose that $\left\{A_{i}\right\}$ is a sequence such that $A=\lim A_{i}$, and for each $i, A_{i} \in(\Delta \cup \Psi)$. Let $I_{1}=\left\{i \mid A_{i} \in \Delta\right\}$ and $I_{2}=\left\{i \mid A_{i} \in \Psi\right\}$. Since $I_{1} \cup I_{2}$ is the set of natural numbers, either $I_{1}$ or $I_{2}$ is infinite. Without loss of generality suppose $I_{1}$ is infinite. Then $\left\{B_{k}\right\}=\left\{A_{i} \mid i \in I_{1}\right\}$ is, in a natural way, a subsequence of $\left\{A_{i}\right\}$, and $B_{k} \in \Delta$ for each $k$, and $A=\lim B_{k}$. Since $\Delta \in \mathfrak{L}$, it follows that $A \in \Delta$. Thus $A \in(\Delta \cup \Psi)$ and we have shown that $(\Delta \cup \Psi) \in \mathscr{L}$. Suppose $\mathcal{F} \subset \mathcal{L}$. It will be shown that $(\bigcap \mathcal{F}) \in \mathcal{L}$. Suppose that $\left\{A_{i}\right\}$ is a sequence of closed subsets of $X, A=\lim A_{i}$, and $A_{i} \in \bigcap \mathcal{F}$. Then for each $\Delta \in \mathcal{F}, A_{i} \in \Delta$. Since $\Delta$ is a limit family, $A \in \Delta$. Thus $A \in \cap \mathcal{F}$. Therefore $\cap \mathcal{F}$ is a limit family.

Theorem 5.3. Let $(X, \Gamma)$ be a topological space and $\left\{A_{i}\right\}$ a sequence of closed subsets of $X$. Then, $\lim A_{i}=A$ if and only if there is an infinite natural number $p$ such that for all $q \geq p, q$ an infinite natural number, ${ }^{0} A_{q}=A$.

Proof. Assume that $\lim A_{i}=A$. Let $s$ be an arbitrary, infinite natural number. Let $x \in{ }^{0} A_{s}$. It will be shown that $x \in A$. For suppose that $x \notin A$. Then there is an open neighborhood $U$ of $x$ such that $U$ intersects only finitely many $A_{i}$. (Otherwise $x$ would be in $\lim \sup A_{i}$ and therefore in $A_{\text {.) }}$ ) Let $t$ be the largest natural number such that $U \cap A_{t} \neq \varnothing$. Then the following statement is true in the standard model:

"if $v$ is a natural number and $v>t$ then $U \cap A_{v}=\emptyset . "$

Thus in the enlargement, ${ }^{*} U \cap A_{s}=\emptyset$. Since $x \in{ }^{0} A_{s}$, let $y$ be such that $y \in A_{s}$ and $x \simeq y$. From the definition of monad it then follows that $y \in{ }^{*} U$. Thus ${ }^{*} U \cap$ $A_{s} \neq \varnothing$, a contradiction. Therefore, $x \in A$ and ${ }^{0} A_{s} \subset A$ for each infinite natural number $s$. Now suppose that $a \in A$. Let $\mathbf{R}(V, W)$ be the following relation:

" $V$ and $W$ are open subsets of $X$ and $a \in W \subset V$."

Then $\mathbf{R}(V, W)$ is a concurrent relation: if $\exists w_{1} \mathbf{R}\left(V_{1}, w_{1}\right), \cdots, \exists w_{n} \mathbf{R}\left(V_{n}, W_{n}\right)$ hold, then $\mathbf{R}\left(V_{1}, W\right), \cdots, \mathbf{R}\left(V_{n}, W\right)$ hold, where $W=\bigcap_{i=1}^{n} V_{i}$. Therefore, in the enlargement, there is a $Z$ such that $\mathbf{R}\left({ }^{*} V, Z\right)$ holds for all open subsets $V$ of $X$ such that $a \in V$. Thus $Z$ is an open subset of ${ }^{*} X$ and $Z \subset \operatorname{monad}$ of $a$. Since $a \in{ }^{*} A$, $a \epsilon^{*} \lim \inf A_{i}$. Thus, in the enlargement, $Z \cap A_{q}=\emptyset$ for all natural numbers $q$ such that $q \geq p$ for some natural number $p$. Without loss of generality, it may be assumed that $p$ is infinite. Thus $a \in{ }^{0} A_{q}$ and $A \subset{ }^{0} A_{q}$. Therefore, there is an infinite $p$ such that for all $q \geq p,{ }^{0} A_{q}=A$.

Assume that $p$ is an infinite natural number and for all $q \geq p,{ }^{0} A_{q}=A$. It will be shown that $A=\lim A_{i}$. Let $x \in A, U$ an open neighborhood of $x$, and $q$ an infinite natural number such that $q \geq p$. Since $x \in{ }^{0} A_{q}$, let $y \in A_{q}$ and such that $x \simeq y$. Then, by the definition of monad, it follows that $y \in{ }^{*} U \cap A_{q}$. That is, ${ }^{*} U \cap A_{q} \neq \emptyset$. Hence the following sentence is true in the enlargement: 
"there is a natural number $n$ such that for all natural numbers $m$, if $m \geq n$ then $* U \cap A_{m} \neq \emptyset$."

Hence in the standard model, by elementary equivalence, it follows that $U \cap A_{i} \neq$ $\emptyset$ for all but finitely many $i$. Since this is true for each open set that contains $x$, $x \in \lim \inf A_{i}$. Thus we have shown that $A \subset \liminf A_{i} \subset \limsup A_{i} \cdot$ In order to show that $\lim \sup A_{i} \subset A$, let $a \in \lim \sup A_{i}$. As before, let $\mathbf{R}(V, W)$ be the concurrent relation: " $V$ and $W$ are open subsets of $X$ and $a \in W \subset V$." Then in the enlargement, there is a $Z$ such that for all open subsets $V$ of $X$ that contain $a$, $\mathbf{R}\left({ }^{*} V, Z\right)$ holds. Thus $Z$ is an open subset of ${ }^{*} X$ and $a \in Z$ and $Z$ is contained in the monad of $a$. Since $a \epsilon^{*} \lim \sup A_{i}$, let $s$ be a natural number that is larger than $p$ and such that $Z \cap A_{s} \neq \emptyset$. Since $a$ is standard, $a \in{ }^{0} A_{s}=A$. Therefore $\limsup A_{i}=\lim \inf A_{i}=A$.

Theorem 5.4. Let $(X, \Gamma)$ be a topological space. Then $\mathcal{C} \subset \mathcal{L}$.

Proof. Let $\Delta \in \mathcal{C},\left\{A_{n}\right\}$ a sequence of members of $\Delta$, and $A=\lim A_{n}$. By Theorem 5.3, there is an infinite natural number $p$ such that $A={ }^{0} A_{p}$. Since $A_{p} \epsilon$ ${ }^{*} \Delta$ and $\Delta \in \mathcal{C},{ }^{0} A_{p}=A \in \Delta$. Therefore, $\Delta \in \mathfrak{Q}$.

Example 5.1. An example of a compact Hausdorff space $(X, \Gamma)$ and a limit family $\Delta, \Delta \subset \Gamma$, such that $\Delta$ is not a compact family. Let $\omega_{1}$ be the first uncountable ordinal, $X=\left\{\alpha \mid \alpha\right.$ is an ordinal and $\left.\alpha \leq \omega_{1}\right\}$, and $\Gamma$ the collection of all subsets of $X$ that are closed in the order topology of $X$. Then $(X, \Gamma)$ is a compact Hausdorff space, and $X$ has the property that if $\left\{a_{n}\right\}$ is a sequence of countable ordinals then there is a countable ordinal $\beta$ such that $\alpha_{n}<\beta$ for each $n$. Thus if $\left\{\alpha_{n}\right\}$ is a sequence of countable ordinals and $\alpha=\lim \alpha_{n}$ then $\alpha$ is a countable ordinal. Let $\Delta=\left\{\{\alpha\} \mid \alpha \in X-\left\{\omega_{1}\right\}\right\},\left\{A_{n}\right\}$ be a sequence of members of $\Delta, A=$ $\lim A_{n}$, and $a_{n}$ such that $\alpha_{n} \in A_{n}$. By Theorem 5.3, let $p$ be an infinite natural number such that $A={ }^{0} A_{p}$. Since $A_{p} \neq \emptyset$ and $(X, \Gamma)$ is compact, ${ }^{0} A_{p}=A \neq \emptyset$. Thus let $\alpha \in A$. Then each neighborhood $U$ of $\alpha$ intersects all except finitely many $A_{n}$; that is, all except finitely many $\alpha_{i}$ are in $U$. Therefore, $\alpha$ is a limit point of the sequence $\left\{\alpha_{i}\right\}$. $\alpha$ is the only such limit point: since if $\beta$ is a limit point of the sequence $\left\{\alpha_{n}\right\}$ and $\beta \neq \alpha$, then, since $(X, \Gamma)$ is a Hausdorff space, there are disjoint neighborhoods $U$ and $V$ of $\alpha$ and $\beta$ respectively, each of wh ich contains all but finitely many $\alpha_{i}-$ which is clearly impossible. Therefore, $\alpha$ is the only member of $A$ and $\alpha=\lim \alpha_{n}$. But then $\alpha$ is countable. Thus $A \in \Delta$ and $\Delta \in \mathcal{L}$. However, $\Delta \notin \mathcal{C}$. For in the enlargement, there is a $\beta \epsilon^{*} X$ such that for each $\alpha \in X-\left\{\omega_{1}\right\}$, $a<\beta<\omega_{1}$. That is, there is a "countable ordinal" $\beta$ in the enlargement that is larger than each standard countable ordinal. It then follows that $\omega_{1} \simeq \beta$. Thus $\{\beta\} \in{ }^{*} \Delta$ and ${ }^{0}\{\beta\}=\omega_{1} \notin \Delta$.

Theorem 5.5. If $(X, \Gamma)$ is a compact metric space, $\Delta \subset \Gamma$, and $\Delta$ is a limit family, then $\Delta$ is a complete family. 
Proof. Suppose $\Delta$ is a limit family. Let $\rho$ be a metric for $(X, \Gamma)$ and $\left\{A_{n}\right\}$ be a Cauchy sequence of members of $\Delta$. Then for some infinite natural number $p$, and all infinite natural numbers $q \geq p,{ }^{0} A_{q}=\lim A_{n}$. Let $A={ }^{0} A_{q}=\lim A_{n}$. Suppose $A \neq \operatorname{Lim} A_{n}$. Then $\lim _{n \rightarrow 0} \rho\left(A_{n}, A\right) \neq 0$. Thus the following statement is true in the standard model: " $r>0$ and for each natural number $m$ there is a natural number $n \geq m$ such that $\rho\left(A_{n}, A\right) \geq r$." Hence in the enlargement for some $s>p$, ${ }^{*} \rho\left(A_{s},{ }^{*} A\right) \geq r$. Since $(X, \Gamma)$ is compact, it follows that $\rho\left({ }^{0} A_{s},{ }^{0}\left({ }^{*} A\right)\right) \geq r$. But since ${ }^{0}\left({ }^{*} A\right)=A$ (Theorem 3.1), $\rho\left({ }^{0} A_{s}, A\right) \geq r$. Since $s>p,{ }^{0} A_{s}=A$. Thus $\rho(A, A) \geq r$-an impossibility. Therefore, $\lim _{n \rightarrow 0}\left(A_{n}, A\right)=0$ and $A=\operatorname{Lim} A_{n}$. Thus $\Delta$ is complete.

Example 5.2. Let $(X, \Gamma)$ be the rational numbers with metric $\rho(x, y)=$ $|x-y|$. Let $\left\{x_{n}\right\}$ be a Cauchy sequence of rational numbers such that $\lim x_{n}=$ $\sqrt{2}$. For each natural number $n$, let $A_{n}=\left\{x_{n}\right\}$. Let $\Delta=\{\emptyset\} \cup\left\{A_{n} \mid n\right.$ is a natural number $\}$. Then $\Delta$ is a limit family that is not a complete family.

Example 5.3. Let $(X, \Gamma)$ be the Euclidean plane and $\Delta$ the family of lines that are contained in $X$. Then, by Example 4.3, $\Delta$ is a complete family. $\Delta$ is not a limit family: since if for each natural number $n, A_{n}$ is the line through $(n, 0)$ parallel to the $Y$-axis, then $\lim A_{n}=\emptyset \notin \Delta$. Thus $\Delta$ is a complete family of a locally compact space that is not a limit family.

Theorem 5.6. Let $(X, \Gamma)$ be a compact metric space and $\Delta \subset \Gamma$. Then the following three statements are equivalent:

(1) $\Delta$ is a compact family,

(2) $\Delta$ is a limit family,

(3) $\Delta$ is a complete family.

Proof. By Theorem 5.4, (1) implies (2); by Theorem 5.5, (2) implies (3); by Theorem 4.8, (3) implies (1).

Definition 5.3. If $\left\{A_{n}\right\}$ is a sequence of closed subsets and there is an $A$ such that $A=\lim A_{n}$, then $\left\{A_{n}\right\}$ is said to be l-convergent. If $\left\{A_{n}\right\}$ are closed subsets of a metric space and there is an $A$ such that $A=\operatorname{Lim} A_{n}$ then $\left\{A_{n}\right\}$ is said to be L-convergent.

The Bolzano-Weierstrass theorem says that in the space $[0,1]$ every sequence of points has a convergent subsequence. Several authors have investigated generalizations of this theorem for sequences of functions and sets. (See [3, p. 340], $[13$, p. 11], [14, p. 124], and [12, p. 229].) For sets, the general version of the Bolzano-Weierstrass theorem has the following form: Every sequence of closed subsets has an l-convergent subsequence. The usual method of proof is to assume the second axiom of countability and construct the limit (to which the correct subsequence will converge) by means of a diagonal argument. Using nonstandard analysis, the problem is in the opposite direction: The limit can be immediately 
found; the problem is to find a subsequence that coverges to it. This method of proof by nonstandard analysis will also allow us to describe exactly those families of subsets that have the Bolzano-Weierstrass property. This method then becomes a powerful tool for finding and proving theorems that involve convergence of subsequences of sequences of closed subsets.

Theorem 5.7. Let $(X, \Gamma)$ be a compact metric space, $\Delta$ a complete family, and $\left\{A_{n}\right\}$ a sequence of members of $\Delta$. Then there is an L-convergent subsequence of $\left\{A_{n}\right\},\left\{B_{i}\right\}$, sucb that $\operatorname{Lim} B_{i}$ is in $\Delta$.

Proof. Let $\rho$ be a metric for $(X, \Gamma)$. Let $p$ be an infinite natural number and $A={ }^{0} A_{p}$. Suppose that no subsequence of $\left\{A_{n}\right\} L$-converges to $A$. A contradiction will be shown. For, in the standard model, the following sentence is then true: "there is a natural number $m$ and a real number $r>0$ such that for all natural numbers $n$ such that $n>m, \rho\left(A_{n}, A\right) \geq r$." Therefore, in the enlargement, ${ }^{*} \rho\left(A_{p},{ }^{*} A\right) \geq r$. Since $(X, \Gamma)$ is compact and ${ }^{0} A_{p}=A$, this is impossible. Thus we have shown that $\left\{A_{n}\right\}$ has an $L$-convergent subsequence, $\left\{B_{i}\right\}$, and $\operatorname{Lim} B_{i}=A$. Since $(X, \Gamma)$ is compact and $\Delta$ is complete, by Theorem 4.8, $\Delta \in \mathcal{C}$. Therefore, since $A_{p} \in{ }^{*} \Delta, \operatorname{Lim} B_{i}=A={ }^{0} A_{p} \in \Delta$.

Example 5.4 (The Blaschke convergence theorem). Let $Y$ be a linear topological space and $X$ a compact subset of $Y$. Suppose that $X$ together with its relative topology $\Gamma$ is a metric space. Then, if $\left\{A_{n}\right\}$ is a sequence of nonempty, closed convex subsets of $X$, then $\left\{A_{n}\right\}$ bas an L-convergent subsequence within $X$ that converges to a nonempty, closed convex subset of $X$.

Proof. Let $\Delta=\{A \mid A$ is a nonempty, closed convex subset of $X\}$. Let $A \in^{*} \Delta$. Then by Example $3.3,{ }^{0} A$ is a closed convex subset of $X$. Since $(X, \Gamma)$ is compact and $A \neq \varnothing,{ }^{0} A \neq \varnothing$. Thus ${ }^{0} A \in \Delta$ and $\Delta$ is a compact family. By Theorem 4.7, $\Delta$ is a complete family. By Theorem 5.7, $\left\{A_{n}\right\}$ has an $L$-convergent subsequence that converges to a member of $\Delta$.

Example 5.5. Let $\Delta_{D_{0},}$ be as in Example 3.2 (the family of all Jordan curves in the closed unit disk of the Euclidean plane that bave area $\geq r$ and that are the image of an affine transformation applied to the Jordan curve $D)$. Let $\left\{A_{n}\right\}$ be a sequence of members of $\Delta_{D . r^{*}}$ Then $\left\{A_{n}\right\}$ bas a subsequence that L-converges to a member of $\Delta_{D, r}$.

Proof. By Example 3.2 and Theorem 4.7, $\Delta_{D_{0},}$ is complete. By Theorem 5.7 , the desired conclusion then follows.

Definition 5.4. Let $(X, \Gamma)$ be a topological space and $\Delta \subset \Gamma . \Delta$ is said to be a Bolzano-Weierstrass family if and only if each sequence of members of $\Delta$ has an $l$-convergent subsequence that converges to a member of $\Delta$. 60 will denote the collection of all Bolzano-Weierstrass families.

In the literature, what is here called Bolzano-Weierstrass families are of ten 
referred to as "compact families," and should not be confused with our use of the term "compact family."

Theorem 5.7. $i d \subset \mathcal{Q}$.

Proof. Let $\Delta \in W$ and $\left\{A_{i}\right\}$ be a sequence of members of $\Delta$ such that $\lim A_{i}=$ $A$. Since $\Delta \in \mathbb{W}$, there is a subsequence of $\left\{A_{i}\right\},\left\{B_{i}\right\}$, such that $\lim B_{i}$ is in $\Delta$. But $\lim B_{i}=A$. Hence $A \in \Delta$ and $\Delta \in \mathfrak{L}$.

Let $(X, \Gamma)$ be a topological space. Unlike $\mathcal{C}$ and $\mathcal{L}, \mathcal{W}$ does not always form a topology on $\Gamma$. In fact, for many interesting spaces, $\Gamma \notin 20$-as is shown by $\mathbb{W}$. Sierpiński in the following theorem of [10]:

Theorem 5.8. Assume the continuum bypothesis. Let $(X, \Gamma)$ be a nonseparable metric space. Then $\Gamma \notin 60$. That is, there is a sequence of closed subsets of $X$ that bas no l-convergent subsequence.

Proof. Since $2^{\boldsymbol{K}_{0}}=\boldsymbol{K}_{1}$, let $\{S\}_{\gamma<\omega_{1}}$ be a well-ordering of all infinite sequences of natural numbers, where $\omega_{1}$ is the first uncountable ordinal. Since $(X, \Gamma)$ is not separable, there is a positive real number, $d$, and a transfinite sequence of points of $X,\left\{x_{\gamma}\right\}_{\gamma<\omega_{1}}$, such that for all $\gamma<\beta<\omega_{1}, \rho\left(x_{\gamma}, x_{\beta}\right) \geq d$, where $\rho$ is the metric for $(X, \Gamma)$. For each natural number $k$, let

$$
E_{k}=\left\{x_{\gamma} \mid \gamma<\omega_{1} \text { and } k \in S_{\gamma}\right\} \text {. }
$$

It will be shown that $E_{0}, E_{1}, E_{2}, \cdots, E_{k}, \cdots$ form a sequence of closed subsets of $X$ that has no convergent subsequence. Since $\rho(x, y) \geq d$, for each $x, y \in E_{k}$, $E_{k}$ is a closed subset of $X$. Let $E_{k_{0}}, E_{k_{1}}, \cdots, E_{k_{n}}, \cdots$ be an arbitrary subsequence of $E_{0}, E_{1}, \cdots, E_{k}, \cdots$. Let $\alpha$ be the countable ordinal such that $S_{\alpha}=$ $\left\{k_{0}, k_{2}, \cdots, k_{2 i}, \cdots\right\}$. Then for each natural number $i, x_{a} \in E_{k_{2 i}}$. Thus $x_{a} \epsilon$ $\lim \sup E_{k_{i}}$. Let $U$ be the open ball about $x_{a}$ of radius $d$. Then, by construction, for each natural number $i, U \cap E_{k_{2 i+1}}=\emptyset$. Thus $x_{a} \notin \liminf E_{k_{i}}$. Therefore $E_{k_{0}}, E_{k_{1}}, \cdots, E_{k_{i}}, \cdots$ is not l-convergent.

Definition 5.5. Let $(X, \Gamma)$ be a topological space. $(X, \Gamma)$ is said to be second countable if and only if there is a sequence of open subsets of $X$ that form a base for the topology on $X$. Such a sequence is called a fundamental system of open subsets of $X$.

Theorem 5.9. If $(X, \Gamma)$ is a second countable topological space then $\mathcal{C}=$ $\mathfrak{W}=\mathfrak{L}$

Proof. (i) Suppose $\Delta \in \mathcal{C}$. Let $\left\{A_{i}\right\}$ be a sequence of members of $\Delta$, $p$ be an infinite natural number, and $A={ }^{0} A_{p}$. Let $\mathcal{F}$ be a fundamental system of open subsets of $X$. Let $V_{0}, V_{1}, \cdots, V_{n}, \cdots$, be those members of $\mathcal{F}$ such that for each $n, V_{n} \cap A \neq \varnothing$; let $W_{0}, W_{1}, \cdots, W_{n}, \cdots$, be those members of $\mathcal{F}$ such that for each $n,{ }^{*} W_{n} \cap A_{p}=\emptyset$. (We are tacitly assuming that there are infinitely many 
members $V$ of $\mathcal{F}$ and infinitely many members $W$ of $\mathcal{F}$ such that $V \cap A \neq \emptyset$ and ${ }^{*} W \cap$ $A_{p}=\varnothing$. Even though this is not always the case, the proof presented here is sufficiently general to allow for the other cases with only slight notational modifications. The reader may prove these additional cases, if he wishes.) Define $B_{n}$ as follows:

$B_{n}$ is the element of the sequence $\left\{A_{i}\right\}$ that has least index and is such that for all $j \leq n, B_{n} \cap V_{j} \neq \varnothing$

and $B_{n} \cap W_{j}=\varnothing$.

We will show that $B_{n}$ exists for each natural number $n$. We first note that it follows from the definition of monad that for each standard natural number $i,{ }^{*} V_{i} \cap$ $A_{p} \neq \emptyset$. Let $n$ be a standard natural number. The statement

$$
\text { "for } i=0, \cdots, n, A_{p} \cap *_{i} \neq \emptyset \text { and } \Lambda_{p} \cap{ }^{*} W_{i}=\emptyset "
$$

is then true in the enlargement. Therefore, the statement

"there is a natural number $m$ such that for $i=1, \cdots, n$,

$$
A_{m} \cap * V_{i} \neq \varnothing \text { and } \Lambda_{m} \cap * W_{i}=\varnothing "
$$

is also true in the enlargement. Thus the statement

"there is a natural number $m$ such that for $i=1, \cdots, n$,

$A_{m} \cap V_{i} \neq \varnothing$ and $A_{m} \cap W_{i}=\varnothing$,

is true in the standard model. The existence of $B_{n}$ immediately follows. Let $x \in A$. Let $V$ be a fundamental open set such that $x \in V$. Then $V=V_{k}$ for some $k$. Hence for all $i \geq k, V \cap B_{i} \neq \emptyset$. Therefore $x \in \lim \inf B_{i}$. Thus $A \subset \liminf B_{i}$. Now let $y \notin A$. Let $S_{0}, S_{1}, \cdots, S_{n}, \cdots$, be the fundamental open sets that conta in $y$. Let $T_{n}=\bigcap_{i=1}^{n} S_{i}$. Let $q$ be an arbitrary, infinite natural number. Then $T_{q} \subset$ monad of $y$. Since $A={ }^{0} A_{p}$, it follows that $T_{q} \cap A_{p}=\emptyset$. Therefore for some stand ard natural number $j,{ }^{*} T_{j} \cap A_{p}=\emptyset$. (Otherwise the set of infinite natural numbers could be shown to be an internal set-which it is not.) Hence for some fundamental open set $W, y \in W$ and ${ }^{*} W \cap A_{p}=\emptyset$. Let $b$ be such that $W=W_{b}$. Then for $i \geq h, B_{i} \cap W_{b}=\emptyset$. Thus $y \notin \limsup B_{i}$. Hence $\limsup B_{i} \subset A$. Since $\limsup B_{i} \subset A \subset \liminf B_{i}, \lim B_{i}=A$. Therefore $\Delta \in(i)$.

(ii) By Theorem $5.7,6 \mathcal{C} \subset \mathcal{L}$.

(iii) Let $\Psi \in \mathcal{L}$ and $D \in{ }^{*} \Psi$. Let $Y_{0}, Y_{1}, \cdots, Y_{n}, \cdots$ be fundamental open subsets of $X$ such that for each natural number $n,{ }^{0} D \cap Y_{n} \neq \varnothing$, and $Z_{0}, Z_{1}, \cdots$, $Z_{n}, \cdots$ be the fundamental open sets such that for each $n, D \cap{ }^{*} Z_{n}=\emptyset$. Let $n$ be an arbitrary, standard natural number. Let $\Psi_{n}=\{E \in \Psi \mid$ for $i=0, \cdots, n, E \cap$ $Y_{i} \neq \emptyset$ and $\left.E \cap Z_{i}=\varnothing\right\}$. Since ${ }^{0} D \cap Y_{i} \neq \emptyset$ for each standard $i$, it follows from the definition of monad that for each standard $i, D \cap{ }^{*} Y_{i} \neq \emptyset$. Thus $D \in{ }^{*} \Psi_{n^{*}}$ Hence in the enlargement ${ }^{*} \Psi_{n} \neq \varnothing$. Therefore $\Psi_{n} \neq \varnothing$. Therefore, by the axiom 
of choice, let $D_{n} \in \Psi_{n}$. Let $t$ be an infinite natural number. We will show that ${ }^{0} D={ }^{0} D_{t}$. Let $v \in{ }^{0} D$. Let $G_{0}, G_{1}, \cdots, G_{m}, \cdots$ be the fundamental open subsets of $X$ that contain $\nu$. For each standard natural number $m$, let $H_{m}=\bigcap_{i=0}^{m} G_{i}$. Then $H_{m}$ is an open subset of $X$ and $v \in H_{m}$. Therefore let $V$ be a fundamental open subset of $X$ such that $v \in V$ and $V \subset H_{m}$. Then $V=V_{k}$ for some standard natural number $k$. Since $D_{t} \cap{ }^{*} V_{k} \neq \varnothing, D_{t} \cap{ }^{*} H_{m} \neq \varnothing$. Since for each standard natural number $m, D_{t} \cap{ }^{*} H_{m} \neq \varnothing$, it follows that there is an infinite natural number $s$ such that $D_{t} r_{1} H_{s} \neq \varnothing$. (Otherwise the set of infinite natural numbers would be internal.) Since $H_{s} \subset$ monad of $v, v \in{ }^{0} D_{t}$. Therefore ${ }^{0} D \subset{ }^{0} D_{t}$. Now assume $z \notin{ }^{0} D$. Then for some open subset $Z$ of $X, z \in Z$ and $Z \cap{ }^{0} D=\emptyset$. Then there is a standard natural number $g$ such that $x \in Z_{g}$ and $Z_{g} \subset Z$. Since $t$ is an infinite natural number, $D_{t} \cap{ }^{*} Z_{g}=\emptyset$. Hence $z \notin{ }^{0} D_{t}$. Therefore ${ }^{0} D_{t} \subset{ }^{0} D$. Thus we have shown ${ }^{0} D={ }^{0} D_{t}$ for each infinite natural number $t$. By Theorem 5.3, $\lim D_{n}={ }^{0} D$. Since $D_{n} \in \Psi$ for each natural number $n$ and $\Psi \in \mathcal{L}, \lim D_{n}={ }^{0} D \in \Psi$. Hence $\Psi \in \mathcal{C}$.

Example 5.6 (The Mahler compactness theorem). Let $(X, \Gamma)$ be Euclidean n. space, $\Delta$ a Mabler family of lattices (Example 3.5), and $\left\{A_{i}\right\}$ a sequence of members of $\Delta$. Then $\left\{A_{i}\right\}$ has an l-convergent subsequence. $\left({ }^{3}\right)$

Proof. By Example 3.5, $\Delta \in \mathcal{C}$. Thus, by Theorem 5.9, $\Delta \in \mathbb{Z}$.

Example 5.7. Let $(X, \Gamma)$ be a second countable, topological group and $\left\{A_{i}\right\}$ a sequence of closed subgroups of $X$. Then $\left\{A_{i}\right\}$ bas a subsequence that locon. verges to a closed subgroup of $X$.

Proof. Let $\Delta$ be the family of closed subgroups of $X$. By Example 3.4, $\Delta \epsilon$ C. Thus by Theorem 5.9, $\Delta \in$ î.

\section{REFERENCES}

1. L. Henkin, Completeness in the theory of types, J. Symbolic Logic 15 (1950), 8191. MR 12, 70.

2. S. T. Hu, Elements of general topology, Holden-Day, London, 1964. MR 31 $\# 1643$.

3. K. Kuratowski, Topology. Vol. 1, Academic Press, New York; PWN, Warsaw, 1966. MR 36 \#840.

4. - Topology. Vol. 2, Academic Press, New York; PWN, Warsaw, 1968. MR 41 \#4467.

5. W. A. J. Luxemburg, A general theory of monads, Internat. Sympos. Applications of Model Theory to Algebra, Analysis, and Probability (Pasadena, Calif., 1967), Holt, Rinehart and Winston, New York, 1969, pp. 18-86. MR 39 \#6244.

6. - A new approach to the theory of monads, Technical Report no. 1, Nonr N00014-66-C0009-A04 (Nr-041-339) for Office of Naval Research, 1967.

7. E. A. Michael, Topologies on spaces of subsets, Trans. Amer. Math. Soc. 71 (1951), 152-182. MR 13, 54. $\# 5680$.

8. A. Robinson, Non-standard analysis, North-Holland, Amsterdam, 1966. MR 34

9. - On some applications of model theory to algebra and analysis, Rend. Mat. e Appl. (5) 25 (1966), 562-592. MR $36 \# 2489$.

$\left({ }^{3}\right)$ A. Robinson in [9] also has a proof of the Mahler compactness theorem by nonstandard analysis. 
10. W. Sierpiński, Sur l'inversion du théorème de Bolzano-Weierstrass généralisé, Fund. Math. 34 (1947), 155-156. MR 9, 83. 62.

11. L. Vietoris, Bereiche zweiter Ordnung, Monatsh. Math. und Phys. 33 (1923), 49-

12. T. Ważewski, Sur les points de division, Fund. Math. 4 (1923), 215-245.

13. G. T. Whyburn, Analytic topology, Amer. Math. Soc. Colloq. Publ., vol. 28, Amer. Math. Soc., Providence, R.I., 1942. MR 4, 86.

14. C. Zarankiewicz, Sur un continu singulier, Fund. Math. 9 (1927), 125-171.

DEPARTMENT OF MATHEMATICS, UNIVERSITY OF CALIFORNIA, LOS ANGELES, CALIFORNIA 90024

Current address: School of Social Sciences, University of California, Irvine, California 92664 